\title{
Manifestações extra intestinais de espectros da doença inflamatória intestinal em crianças e adolescentes: artigo de revisão
}

\section{Extra intestinal manifestations of spectros of intestinal inflammatory disease in children and adolescents: article of review}

Mara Larissa Alves Marques ${ }^{1}$. Marcos Paulo Fernandes Patrício².

1 Médica, Residência em Pediatria, Hospital Universitário Walter Cantídio (HUWC), Título de Especialista em Pediatria, Sociedade Brasileira de Pediatria (SBP), Fortaleza, Ceará, Brasil. 2 Médico, Residente de Pediatria, Hospital Universitário Walter Cantídio (HUWC), Fortaleza, Ceará, Brasil.

\section{RESUMO}

A doença inflamatória intestinal (DII) caracteriza-se pela inflamação crônica e idiopática do trato gastrointestinal que incluem doença de Chron (DC), retocolite ulcerativa (RCU) e as colites indeterminadas (CI); nas crianças, que correspondem a cerca de $10 \%$ dos casos nos EUA, há comumente história familiar de DII, sugerindo associação genética. Fatores ambientais também estariam relacionados como fatores de risco ou protetores. Geralmente os pacientes pediátricos apresentam quadros iniciais mais graves e extensos da doença, como comprometimento nutricional, atraso de crescimento e puberdade, bem como maior prevalência de sintomas extra intestinais. Podem ser de apresentações e gravidade variáveis, acometendo olhos, rins, articulações, dentre outros. Este estudo se propõe a descrever as principais manifestações extra intestinais mais comuns em pacientes com doença inflamatória intestinal na população pediátrica, a partir de revisão de literatura.

Palavras-chave: Doença de Chron. Colite ulcerativa. Criança. Adolescente.

\begin{abstract}
Inflammatory bowel disease (IBD) is characterized by chronic idiopathic inflammation of the gastrointestinal tract including Crohn's disease (CD), ulcerative colitis (UC) and indeterminate colitis (IC); in children, which correspond to about $10 \%$ of the cases in the U.S., there are commonly family history of IBD, suggesting genetic association. Environmental factors are also related risk or protective factors. In general pediatric patients have more severe initial frames and extensive disease, as nutritional impairment, delayed growth and puberty, as well as a higher prevalence of extra intestinal symptoms. These manifestations can be of variable presentations, as well as its severity, affecting the eyes, kidneys, joints, among others. This study aims to describe the main extra intestinal manifestations more common in patients with inflammatory bowel disease in the pediatric population, through a literature review.
\end{abstract}

Keywords: Crohn's disease. Ulcerative colitis. Child. Adolescent.

Autor correspondente: Mara Larissa Alves Marques, Rua Bruno Porto, 555, Parque Iracema, Fortaleza, Ceará. CEP: 60824-010. Telefone: +55 85 99403-7412. E-mail: maralarissam@gmail.com

Conflito de interesses: Não há qualquer conflito de interesses por parte de qualquer um dos autores.

Recebido em: 28 Ago 2017; Revisado em: 07 Dez 2017; Aceito em: 08 Jan 2018. 


\section{INTRODUÇÃO}

Estima-se que mais de um milhão de pessoas nos Estados Unidos tem doença inflamatória intestinal (DII) e cerca de $10 \%$ destes pacientes são crianças. ${ }^{1}$ Representa um espectro de distúrbios que incluem retocolite ulcerativa (RCU), doença de Crohn (DC) e colites indeterminadas (CI), com inflamação crônica e idiopática do trato gastrintestinal (Tabela 1). O tipo de DII geralmente é determinada pela parte do trato gastrointestinal (TGI) que é afetada. ${ }^{2} \mathrm{~A}$ doença de Chron acomete qualquer parte do TGI, da boca ao ânus, e transmural. A inflamação associada a RCU usualmente se inicia no reto e estende-se proximalmente, com um padrão de continuidade; eventualmente pode acometer todo o cólon. Quando não se pode firmar diagnóstico de RCU ou DC a partir de critérios clínicos, radiológicos, endoscópicos ou histopatológicos, a criança com doença intestinal é descrita como colite indeterminada. ${ }^{3}$ Embora ambas tenham patologias e características clínicas, sua patogênese ainda é pobremente compreendida. A proporção de colite indeterminada diminui com o aumento da idade, sugerindo que a colite indeterminada deve representar uma forma de acometimento de DII que se apresenta antes do fenótipo definitivo ou que os critérios de classificação atual não seriam aplicáveis a crianças menores.

Tabela 1. Pacientes com diagnóstico de doença inflamatória intestinal na infância.

\begin{tabular}{lccc}
\hline $\begin{array}{l}\text { Crianças com } \\
\text { DII (n=416) }\end{array}$ & DC & RCU & CI \\
\hline $\mathrm{N}$ & 276 & 99 & 41 \\
$\begin{array}{l}\text { Masculino/ } \\
\text { Feminino }\end{array}$ & $164 / 112$ & $48 / 51$ & $19 / 22$ \\
$\begin{array}{l}\text { Idade média } \\
\text { ao diagnóstico }\end{array}$ & $11.6(8.9-13.2)$ & $10.9(8.8-10.8)$ & $10(7.6-12.5)$ \\
$\begin{array}{l}\text { Caucasianos } \\
\begin{array}{l}\text { Duração média } \\
\text { do seguimento } \\
\text { (anos) }\end{array}\end{array}$ & $97.8 \%(270 / 276)$ & $94.9 \%(94 / 99)$ & $97.5 \%(40 / 41)$ \\
\hline
\end{tabular}

Fonte: Adaptada de Van Limbergen J, Russell RK, Drummond HE, Aldhous MC, Round NK, Nimmo ER. Definition of phenotypic characteristics of childhood-onset inflammatory bowel disease. Gastroenterology. 2008;135(4):1114.

Uma distribuição bimodal tem sido vista, com um início precoce entre 10-20 anos de idade, e outra, mas com um pico menor, entre 50-80 anos. Aproximadamente 25\% dos pacientes apresentam antes dos 20 anos. A DII pode ser tão precoce como no primeiro ano de vida, e esse aumento de incidência entre crianças jovens tem sido observada desde a virada do século. Crianças com início precoce de DII tem mais frequentemente o envolvimento do cólon. Nos países desenvolvidos, esses distúrbios são as maiores causas de inflamação intestinal crônica. ${ }^{4}$

A maioria dos pacientes pediátricos são diagnosticados na infância tardia e adolescência, embora observe-se
precocemente.Aincidência de DII em crianças está em aumento. Um estudo canadense, relatou um aumento de 21,3 para 26,2 por 200 habitantes entre 1999 e 2008, com o crescimento mais evidente em indivíduos com menos de 10 anos de idade. $\mathrm{Na}$ Finlândia sugeriu-se que a incidência de DII dobrou entre os anos de 1987 e 2003, um período de 15 anos. ${ }^{5}$ As crianças, em geral, apresentam quadros iniciais mais graves e extensos da doença, maior prevalência de manifestações extra intestinais (Quadro 1) e importante comprometimento nutricional, além de particularidades como atraso do crescimento e do desenvolvimento, quando comparadas aos adultos. Tais fatos podem ser decorrentes do controle inadequado da inflamação, do retardo no diagnóstico, da resposta imune, de fatores ambientais, dietéticos, do microbioma, das manifestações extra intestinais e da dificuldade de se obter a manutenção da remissão ou ainda dos efeitos colaterais do tratamento. ${ }^{6}$

Quadro 1. Manifestações extra intestinais na DII.

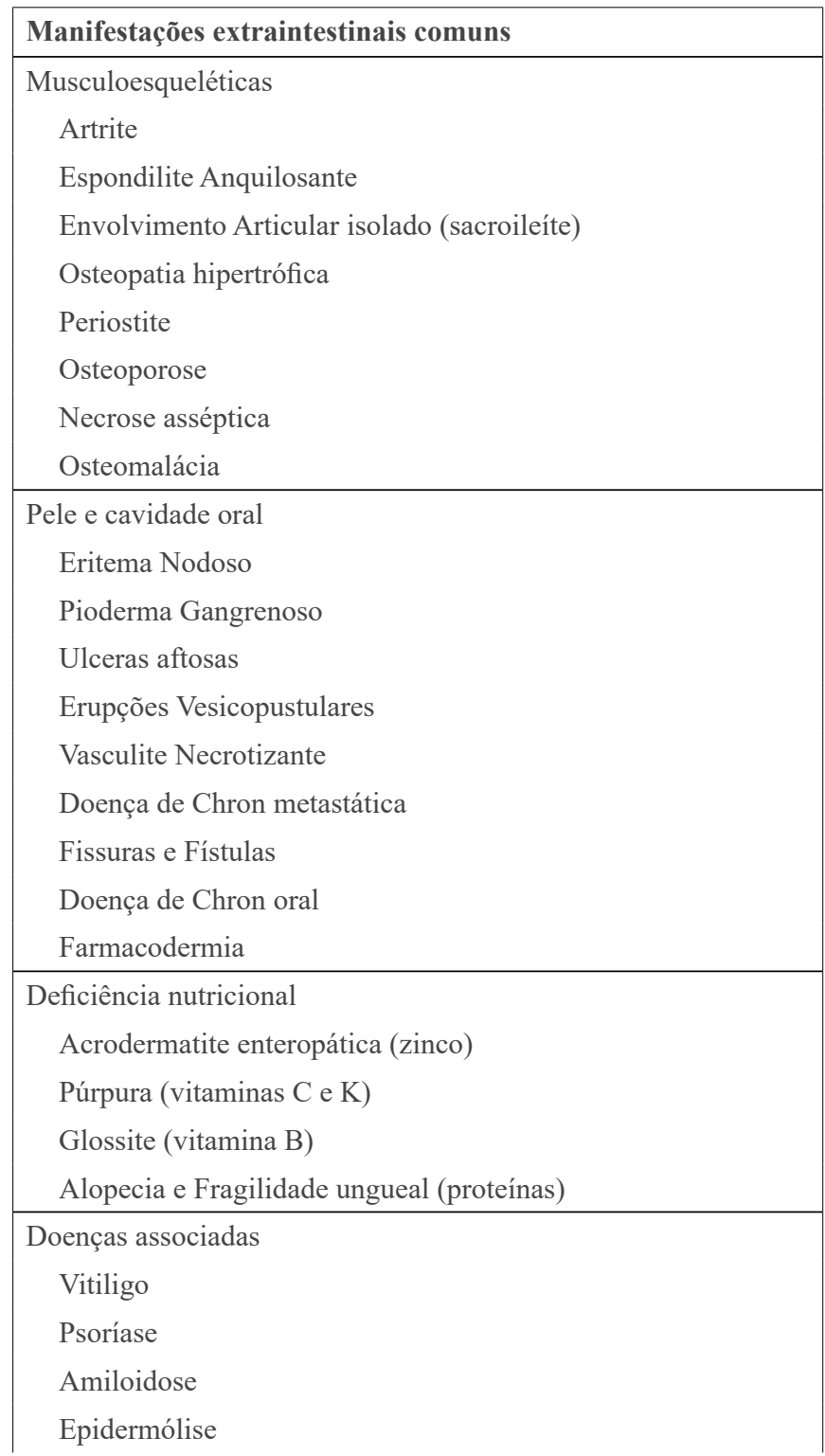

Continua. 
Continuação.

Quadro 1. Manifestações extra intestinais na DII.

\begin{tabular}{|l|}
\hline Manifestações extraintestinais comuns \\
\hline Hepatobiliar \\
Colangite Esclerosante Primária \\
Colangite Esclerosante Primária de Pequenos Ductos \\
Carcinoma de Ductos Biliares \\
Colelitíase \\
Hepatite Crônica Autoimune \\
Pericolangite \\
Fibrose Portal \\
Esteatose Hepática \\
\hline
\end{tabular}

Oftalmológicos

Uveíte

Iriti

Episclerite

Escleromalácia

Úlceras de Córnea

Retinopatia

Neurite Retro bulbar

Ceratopatia do Chron

Metabólicos

Retardo do Crescimento

Atraso Puberal

Manifestações extraintestinais menos comuns

Hematológicas e vasculares

Anemia- Ferropriva, por Deficiência de Folato ou Vitamina B12, Hemolítica, da Doença Crônica

Púrpura Trombocitopênica

Tromboflebite

Tromboembolismo

Arterite

Poliarterite Nodosa

Arterite de Takayasu

Anticorpo Anti cardiolipina

Vasculite Cutânea

Hipoesplenismo

Renal e trato genitourinário

Cálculo Renal (cálculos de oxalato na doença ileal)

Extensão local da DC envolvendo ureter ou bexiga

Amiloidose

Nefrotoxicidade relacionada a Drogas

Dano Tubular Renal

Neurológico

Neuropatia periférica

Mielopatia

Disfunção Vestibular

Continua.
Conclusão.

Quadro 1. Manifestações extra intestinais na DII.

\begin{tabular}{|l|}
\hline Manifestações extraintestinais comuns \\
\hline Pseudotumor Cerebral \\
Miastenia Gravis \\
Trato respiratório \\
Fibrose Pulmonar \\
Vasculite \\
Bronquite \\
Laringotraqueíte aguda \\
Doença intersticial \\
Sarcoidose \\
\hline
\end{tabular}

Crianças também comumente apresentam história familiar de DII, sugerindo forte associação genética. ${ }^{7}$ Em estudo realizado no Reino Unido, foram recrutados de todos os centros de gastroenterologia pediátrica na Escócia 416 pacientes com diagnóstico de DII antes do $17^{\circ}$ aniversário. Observou-se que 276 destes foram classificados com DC, 99 como RCU e 41 como colite indeterminada. ${ }^{8}$

Dados são controversos no que diz respeito a relação doença de Chron e retocolite ulcerativa e aleitamento. O aleitamento estimula a maturação e desenvolvimento da mucosa intestinal, protegendo de infecções gastrointestinais na infância. Estudos sugerem que DII acometeria menos frequentemente crianças amamentadas. Sugere-se ainda que exposição a infecções no período neonatal, como diarreias e a exposição precoce a antibióticos poderiam aumentar o risco de DII, sendo o risco ainda maior se a exposição for antes dos primeiros anos de vida. ${ }^{9}$ A exposição precoce a animais teria um efeito protetor. A observação tem a hipótese de que a exposição a patógenos precocemente conferiria ao indivíduo proteção contra doenças imunomediadas. ${ }^{10}$ Quanto à dieta, há sugestão de que o aumento da ingesta de gorduras (mono ou poli-insaturado) estão relacionadas positivamente a DII, enquanto uma dieta rica em fibras e vitamina $\mathrm{D}$ teriam uma relação inversa. ${ }^{11-13}$

A resposta imunológica já foi identificada como crucial para DII (doença inflamatória intestinal). Isso é influenciado tanto por fatores humorais quanto microbiológicos, que interagem, podendo resultar em reposta exagerada com consequente lesão da mucosa. ${ }^{14}$ Defeitos em qualquer desses aspectos do sistema imunológico resultam em resposta anormal (excessiva ou ineficaz) à microbiota intestinal. São eles: Defeito na barreira epitelial, ${ }^{15}$ disfunção da resposta celular ${ }^{16}$ ou níveis anormais de fatores humorais nos tecidos: inflamatórios ou imunorreguladores. ${ }^{17}$ A flora intestinal parece também estar implicada no desenvolvimento de DII quando associada a defeitos imunogênicos. ${ }^{18}$ Embora a flora constitua um fator importante, não foi possível identificar um agente intimamente relacionado a DII. Em crianças, é possível identificar alterações na população da microbiota já na instalação da DII. ${ }^{19}$ Vários loci genéticos foram relacionados a DII. Alguns estão diretamente ligados à doença. Outros atuam apenas na modulação de expressão proteica, atuando de forma conjunta 
para determinar maior risco para o desenvolvimento da DII. Essas alterações genéticas atingem vias relacionadas à doença inflamatória intestinal, e podem somar-se determinando uma instalação precoce da doença. Essas vias imunológicas comprometidas podem estar relacionadas a outras doenças imunomediadas ou conferir alguma imunodeficiência. ${ }^{18}$

Pacientes com DII tipicamente apresentam manifestações na idade escolar (avaliar mudança para escolar) e adolescência, e podem apresentar uma ou várias das manifestações: sintomas gastrointestinais (diarreia, sangue nas fezes, dor abdominal), distúrbios de crescimento (déficitde crescimento, atraso puberal, desnutrição ou mesmo sobrepeso), além de manifestações extra intestinais (estomatite aftosa, eritema nodoso, pioderma gangrenoso, uveíte, icterícia ou hepatomegalia, artrite).

A suspeita de DII parte da combinação de dados clínicos e de exames complementares, incluindo exames radiológicos e endoscópicos, incluindo biópsias. Essa investigação ajuda a localizar a doença e fazer a distinção em DC ou RCU. Nenhum parâmetro clínico e laboratorial é útil em predizer isoladamente a gravidade dessas doenças em crianças. Especificamente na DC, foi criado um escore, denominado PCDAI (Pediatric Crohn's Disease Activity Index - Quadro 2), com o objetivo de avaliar a gravidade da doença, com grande utilidade no acompanhamento clínico das crianças. Um escore maior que 30 ocorre na doença moderada a grave, o que sinaliza para a necessidade de uma terapêutica mais agressiva. Inclui 4 parâmetros: história, exame físico, antropometria e testes laboratoriais..$^{20}$ Já na RCU, utilizase o PUCAI (Pediatric Ulcerative Colitis Activity Index - Quadro 3), o qual utiliza apenas sintomas relatados pelos pacientes. $\mathrm{O}$ escore é dividido em doença sem atividade $(<10)$, atividade leve (10-34), atividade moderada (35-64) e atividade grave ( $>$ ou igual a 65). Existem algumas limitações nesse escore, não devendo ser utilizado em pacientes com doença restrita ao reto, além disso, ele não é capaz em diferenciar a colite fulminante da colite grave. ${ }^{21}$

Quadro 2. Índice de atividade da doença de Crohn em crianças.

\begin{tabular}{|lc|}
\hline Anamnese & \\
\hline Dor abdominal & 0 \\
Ausente & 5 \\
Leve- não interfere nas atividades diárias & 10 \\
Moderada a Grave- Diária, Noturna ou afeta atividades diárias & \\
\hline Evacuações (vezes/dia-aspecto) & 0 \\
0-1, líquidas, sem sangramento & 5 \\
$>2$ pastosas com pequeno sangramento ou 2-5 líquidas & 10 \\
Sangramento importante ou $\geq 6$ líquidas ou diarreia noturna & \\
\hline $\begin{array}{l}\text { Estado geral do paciente } \\
\text { Bem, sem limitações das atividades diárias }\end{array}$ & 0 \\
$\begin{array}{l}\text { Dificuldade ocasional em desempenhar atividades } \\
\text { adequadas para idade }\end{array}$ & 5 \\
Limitações frequentes de atividades & 10 \\
\hline
\end{tabular}

Continua.
Continuação.

Quadro 2. Índice de atividade da doença de Crohn em crianças.

\begin{tabular}{|c|c|c|c|c|}
\hline \multicolumn{5}{|c|}{ Laboratório } \\
\hline \multicolumn{5}{|c|}{ Hematócrito (\%) } \\
\hline 10 anos & \multicolumn{4}{|c|}{$11-14 \mathrm{a} / \mathrm{M}$} \\
\hline$>33$ & 0 & $>35$ & \multicolumn{2}{|l|}{0} \\
\hline $28-32$ & 2.5 & $30-34$ & \multicolumn{2}{|c|}{2.5} \\
\hline$<28$ & 5 & $<30$ & \multicolumn{2}{|l|}{5} \\
\hline $11-19$ a $/ F$ & \multicolumn{4}{|c|}{$15-19$ a $/ \mathrm{M}$} \\
\hline$>34$ & 0 & $>37$ & \multicolumn{2}{|l|}{0} \\
\hline $29-33$ & 2.5 & $32-36$ & \multicolumn{2}{|l|}{2.5} \\
\hline$<29$ & 5 & $<32$ & \multicolumn{2}{|l|}{5} \\
\hline \multicolumn{5}{|l|}{ Vhs } \\
\hline \multicolumn{3}{|l|}{$<20$} & & 0 \\
\hline \multicolumn{3}{|l|}{$20-50$} & & 2.5 \\
\hline \multicolumn{3}{|l|}{$>50$} & & 5 \\
\hline \multicolumn{5}{|l|}{ Albumina } \\
\hline \multicolumn{3}{|l|}{$\geq 3.5$} & & 0 \\
\hline \multicolumn{3}{|l|}{$3.1-3.4$} & & 2.5 \\
\hline \multicolumn{3}{|l|}{$\leq 3.0$} & & 5 \\
\hline \multicolumn{5}{|c|}{ Exame físico } \\
\hline \multicolumn{5}{|l|}{ Peso } \\
\hline \multicolumn{4}{|c|}{ Ganho de Peso/Peso estável/ Perda voluntária de Peso } & 0 \\
\hline \multicolumn{4}{|c|}{ Perda ou Manutenção Involuntária de Peso (1-9\%) } & 2.5 \\
\hline \multicolumn{4}{|c|}{ Perda Involuntária de Peso $>10 \%$} & 5 \\
\hline \multicolumn{5}{|c|}{ Estatura ao diagnóstico } \\
\hline \multicolumn{3}{|c|}{ <1 Desvio Padrão (DP) } & & 0 \\
\hline \multicolumn{3}{|l|}{$1-2 \mathrm{DP}$} & & 5 \\
\hline \multicolumn{3}{|l|}{$>2 \mathrm{DP}$} & & 10 \\
\hline Seguiment & & & & \\
\hline Velocidade & scime & & & 0 \\
\hline Velocidade & scime & $E>-2$ DP & & 5 \\
\hline Velocidade & scime & & & 10 \\
\hline Exame ab & & & & \\
\hline Sem desco & u mas & & & 0 \\
\hline Desconfort & Iassa s & forto & & 5 \\
\hline Desconfort & sa Inv & u Massa Definida & & 10 \\
\hline Doença pe & & & & \\
\hline Ausente & & & & 0 \\
\hline $\begin{array}{l}\text { 1-2 Fístul } \\
\text { Desconfort }\end{array}$ & intom & nagem Escassa, & & 5 \\
\hline Fístula Ati & hagem & to ou Abscesso & & 10 \\
\hline
\end{tabular}

Continua. 
Conclusão.

Quadro 2. Índice de atividade da doença de Crohn em crianças.

\begin{tabular}{|l|}
\hline Manifestações extra intestinais \\
(Febre $\geq 38.5^{\circ} \mathrm{C}$ por 3 dias na última semana, Artrite, \\
Uveíte, Eritema Nodoso, Pioderma Gangrenoso) \\
Nenhum \\
Um \\
$\geq$ Dois \\
\hline Pontuação total \\
\hline
\end{tabular}

Legenda: VHS: Velocidade de hemossedimentação; M: Masculino; F: Feminino

Fonte: Adaptado de Hyams JS, Ferry GD, Mandel FS, Gryboski JD, Kibort PM, Kirschner BS, et al. Development and validation of a pediatric Crohn's disease activity index. J Pediatr Gastroenterol Nutr. 1991;12(4):439-47.

Quadro 3. Índice de atividade de retocolite ulcerativa em crianças.

\begin{tabular}{|clc|}
\hline Item & & Pontuação \\
\hline 1. & Dor abdominal & \\
& Ausente & 0 \\
& Pode ser ignorada & 5 \\
& Não pode ser ignorada & 10 \\
\hline
\end{tabular}

\section{Sangramento retal}

Ausente

0

Pequeno, presente em $<50 \%$ das evacuações

Pequeno, presente na maioria das evacuações

Volumoso

30

3. Consistência das fezes

Sólidas

0

Pastosas

5

Aquosas

10

4. Número de evacuações em 24 h

$0-2$

0

3-5

5

6-8

10

$>8$

15

5. Evacuações noturnas

Sim

Não

10

6. Nível de atividades

Sem limitações de atividades 0

Limitação ocasional de atividades

Limitação importante de atividades

Fonte: Adaptado de Turner D, Otley AR, Mack D, Hyams J, de Bruijne J, Uusoue K, et al. Development, validation, and evaluation of a pediatric ulcerative colitis activity index: a prospective multicenter study. Gastroenterology. 2007;133(2):423-32.

O quadro clínico depende, primariamente, da localização e extensão das lesões, além da idade da apresentação da doença. Nas crianças menores de cinco anos, que apresentam mais envolvimento do intestino grosso, o sintoma mais observado é a diarreia com sangue e dor abdominal em até 91\% dos pacientes, podendo ser observado lesões perianais. ${ }^{22}$

A RCU é caracterizada por uma inflamação difusa das camadas mucosa e submucosa, que não se aprofunda na parede da mucosa, limitada ao colón, que geralmente se inicia no reto e avança cranialmente, de forma contínua. A doença pode ser classificada pela extensão do acometimento da mucosa. De acordo com a Classificação de Montreal, essa patologia é considerada proctite, quando está confinada ao reto, colite esquerda, com acometimento até a flexura esplênica, e colite extensa, quando envolve além do ângulo esplênico. Na população pediátrica tem um quadro clínico semelhante ao encontrado em adultos. Devido ao intenso quadro inflamatório na mucosa, os sintomas mais prevalentes são sangramento retal, diarreia, dor abdominal, tenesmo e urgência fecal. ${ }^{23}$

A colite indeterminada é um termo utilizado para os pacientes que apresentam características clínicas, endoscópicas e histológicas compatíveis com o diagnóstico de doença de Crohn e Retocolite Ulcerativa, não permitindo a diferenciação entre essas duas patologias. Cerca de $10 \%$ dos casos são classificados com CI. Os sistemas de classificação mais novos recomendam que o termo colite indeterminada seja reservado para os pacientes que permaneçam indefinidos após a colectomia e análise histológica. Os demais deverão ser considerados DII não classificada. ${ }^{21}$

Sintomas extra intestinais podem surgir em diversas partes do corpo, acometendo olhos, rins, articulações, pele, ossos, fígado. ${ }^{3}$ Déficit de crescimento e consequente atraso puberal também são uma manifestação comum de DII. ${ }^{24}$ Pode haver ainda desnutrição, deficiências específicas de micronutrientes, a até, eventualmente, sobrepeso e obesidade. Estas condições também determinam vários problemas psicossociais e econômicos, falta ao trabalho e escola, depressão, alteração de imagem corporal e baixa autoestima, dificuldade de socialização e sexualidade, que limitam a qualidade de vida. ${ }^{4}$ $\mathrm{O}$ presente artigo tem por objetivo realizar revisão sobre as principais repercussões extra intestinais em indivíduos portadores de DII, na população pediátrica.

\section{METODOLOGIA}

Para elaboração do presente estudo, foi realizada revisão sistemática na bases de dados PUBMED, Scielo, LILACS, Clinical Key, buscando a literatura publicada, do período de janeiro de 2005 a junho de 2017 com os seguintes termos: doença inflamatória intestinal E crianças OU adolescentes OU pediatria; manifestações extra intestinais da doença inflamatória intestinal em crianças OU pediatria inflamatory bowel disease AND children OR pediatrics; inflamatory bowel disease AND extra intestinal symptoms. Foram selecionados 39 artigos e excluídos os artigos relacionados a apresentação clínica em adultos. Após interpretação dos artigos e capítulos, organizou -se os temas em grupos. Não foi necessária apreciação do Comitê de Ética por se tratar de uma revisão. 


\section{MANIFESTAÇÕES EXTRA INTESTINAIS}

Apesar do seu nome, a DII não é limitada ao intestino e cerca de $30 \%$ dos pacientes desenvolverão uma manifestação extra gastrointestinal precedente ou na evolução da doença. ${ }^{25}$

Manifestações extra intestinais associadas a DII podem incluir entre 10 a $30 \%$ dos pacientes nos primeiros anos de diagnóstico. Podem ser acometidos cavidade oral, pele, articulações, fígado, olhos e, raramente, outros órgãos (Tabela 1). Os pacientes podem manifestá-las isoladamente ou, mais comumente, associada a doença do cólon. Essas manifestações extra intestinais são mais comuns na DC em relação a RCU, mas não estão firmemente relacionados a atividade da doença. Ocasionalmente, pacientes podem apresentar tais sintomas antes de uma apresentação intestinal evidente. ${ }^{26,27}$

\section{LESÕES ORAIS}

Uma variedade de manifestações na cavidade oral pode ocorrer. São relatadas lesões específicas e não específicas. Estomatite aftosa ocorrem em cerca de $10 \%$ das crianças com DII, e está associada cerca de três vezes mais a DC que a RCU. Os achados associados incluem edema labial, gengival e de mucosa. ${ }^{23}$ Muitos consideram como lesões inespecíficas, uma vez que $20 \%$ da população geral pode apresentá-la; no entanto, na DII, a estomatite aftosa apresenta lesões mais extensas e persistentes (Figura 1).

Granulomatose orofacial é uma manifestação comum em crianças com DII e pode ser a primeira manifestação clínica da DC e é caracterizada por edema labial, na face, gengival ou mucosa oral com aspecto de granuloma não caseoso ao exame histológico. Os lábios apresentam aumento de volume difuso e localizado, de consistência borrachosa, que não regridem, ao contrário do angioedema; o tecido é firme e sensível quando palpado (Figura 2). Os achados orais precedem os sintomas intestinais em $50 \%$ dos casos. ${ }^{28}$

Por outro lado, Pioestomastite vegetante (Figura 3) é mais característico de RCU, caracterizada por múltiplas pústulas, erosões e úlceras em uma placa eritematosa difusa com vegetações na mucosa. ${ }^{29}$

Figura 1. Estomatite aftosa.

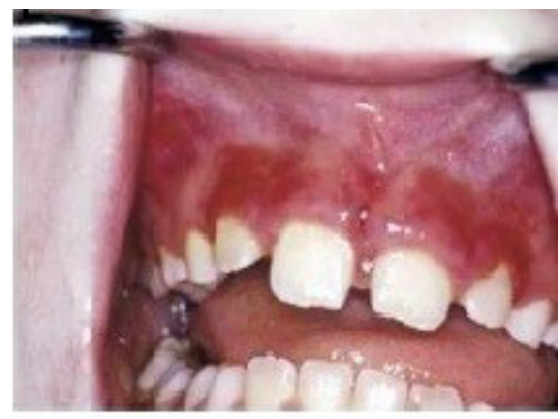

Fonte: Woo SB. Atlas de patologia oral. Rio de Janeiro: Elsevier; 2013.
Figura 2. Granulomatose orofacial.

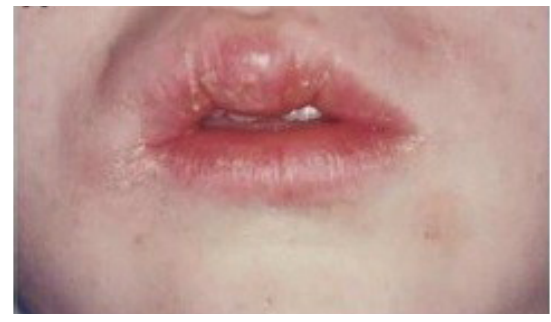

Fonte: Woo SB. Atlas de patologia oral. Rio de Janeiro: Elsevier; 2013.

Figura 3. Pioestomatite vegetante.

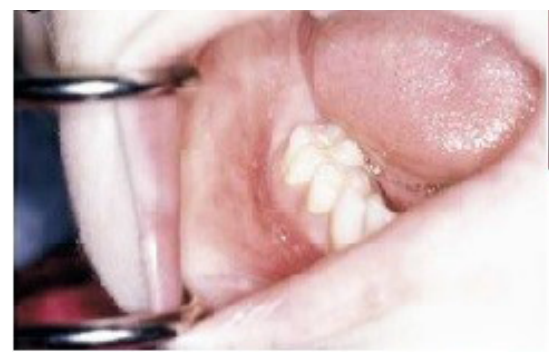

Fonte: Woo SB. Atlas de patologia oral. Rio de Janeiro: Elsevier; 2013.

\section{LESÕES DERMATOLÓGICAS}

Eritema nodoso (Figura 4) é a manifestação dermatológica mais comum na DII, caracterizado por lesões de 1 a $3 \mathrm{~cm}$ de diâmetro, dolorosas, vistas com maior frequência na tíbia. Está presente em cerca de 5\% das crianças com DII e apresenta resolução rápida quando instituído o tratamento adequado da DII. ${ }^{30}$ Acomete tanto pacientes com DC como com RCU. Pode também ser causado por outras patologias, como infecções (Estreptococos de grupo A, tuberculose), medicações como Penicilina ou Fenitoína, doenças autoimunes (Artrite Reumatoide, Lúpus) e neoplasias. Pioderma gangrenoso, embora seja a segunda manifestação dermatológica nos pacientes com DII, é raro em crianças e é mais observado em pacientes com RCU que em DC (no entanto também descrito nesses pacientes); trata-se de importante rash ulcerado que responde a imunossupressão.

Figura 4. Eritema nodoso.

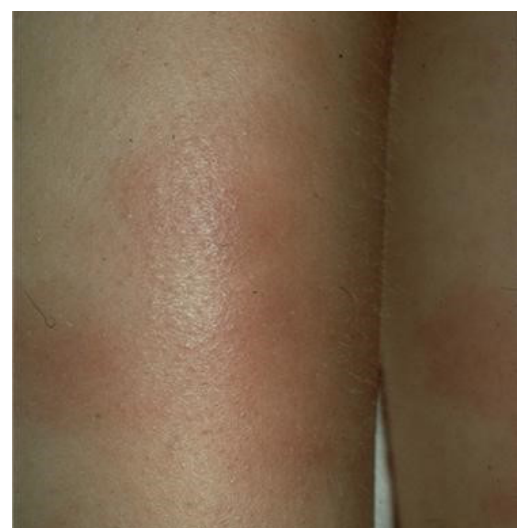

Fonte: DermIS. Erythema nodosum. Disponível em: http://www.dermis.net/ dermisroot/en/30507/image.htm. 


\section{ACOMETIMENTO OCULAR}

Dados sobre as manifestações oculares da doença inflamatória intestinal (IBD) em crianças são limitados. Alguns autores relataram uma alta prevalência de uveíte assintomática, ainda a importância destas observações é desconhecida e não há nenhuma recomendação em que deve ser oferecido acompanhamento oftalmológico. ${ }^{31}$

Manifestações oculares comuns associadas com DII incluem conjuntivite, episclerite, blefarite, esclerite, uveíte, catarata, ceratopatia, papillite, vasculite, neurite óptica, miosite orbital e pseudotumor orbital. Fora estes, episclerite, esclerite e uveíte são as manifestações oculares mais comuns em adultos DII. Embora as manifestações oculares ocorram geralmente no início do curso da doença, é raro ter manifestação ocular precedendo o diagnóstico de DII. ${ }^{32,33}$

\section{ACOMETIMENTO ARTICULAR}

Em relação ao acometimento articular, cerca de 4\% dos pacientes com DII apresentam artrite nos primeiros anos de diagnóstico, enquanto mais de 17\% apresentam artralgia. A artrite é mais comum na DC que na RCU, embora seja mais evidente na colite de Chron que no paciente com colite gastroduodenal. ${ }^{26,27}$ Estudos prévios já demonstraram que sintomas como artralgia, artrites e o eritema nodoso, tendem a ser mais evidentes nos períodos de atividade da doença. ${ }^{27}$ Trata-se de uma artrite tipicamente não erosiva que afeta grandes articulações. A ocorrência de osteopenia e osteoporose podem ser justificadas pelo distúrbio da absorção de cálcio e vitamina $\mathrm{D}$, desnutrição, exposição a corticoides, atividade da doença e elevação nos níveis de citocinas, embora sua patogênese não seja completamente compreendida. ${ }^{26}$

\section{HEPATOBILIAR}

Pacientes com DII podem apresentar elevação inespecífica das transaminases tanto pela esteatose hepática como pelo tratamento. Os pacientes podem apresentar fadiga, prurido e icterícia intermitente. Sua gravidade não está relacionada a atividade da DII. O diagnóstico é feito pela combinação entre imagem (ultrassom, ressonância magnética, colangeopancreatografia retrógrada) e biópsia hepática. O tratamento é feito com ácido ursodesoxicólico. ${ }^{34}$

Colangite esclerosante primária (CEP) e hepatite autoimune (HAI) são as principais doenças hepáticas imune mediadas que ocorrem em crianças além da infância. Ambas as doenças ocorrem em uma maior frequência em pacientes com doença inflamatória intestinal (DII). A CEP é principalmente uma doença colestática caracterizada por inflamação e fibrose periductais dos ductos biliares intra ou extra-hepáticas, considerando que a HAI é caracterizada por inflamação do trato portal que pode se estender para o lóbulo hepático. Muitos pacientes, especialmente crianças, têm sobreposição, com características de ambas as doenças, e isto é denominado colangite esclerosante autoimune (ASC). Tanto CEP e HAI podem progredir para hipertensão portal e cirrose e, finalmente, necessitam de transplante de fígado. ${ }^{34}$

\section{ESTADO NUTRICIONAL E METABÓLICO}

Pacientes pediátricos com DII, principalmente aqueles portadores de DC, apresentam alteração no estado nutricional e a desnutrição é frequentemente relatada. Há uma propensão para o baixo peso em pacientes com DII, mas os estudos mostraram algumas mudanças nesse perfil, como aumento nos últimos anos do número de portadores de sobrepeso ou obesidade, principalmente naqueles com RCU. Com o advento dos tratamentos para DII, a desnutrição e o baixo peso podem deixar de ser um marcador de gravidade das DII, passando as crianças a terem altas taxas de sobrepeso e obesidade, assim como a população geral. ${ }^{4} \mathrm{O}$ retardo de crescimento é comum em pacientes com diagnóstico de DII na infância e este pode ser o único sintoma. ${ }^{35}$ Deficiências de nutrientes e vitaminas são relatadas, principalmente a deficiência de vitamina D. ${ }^{36}$ Os comprometimentos nutricionais relatados foram mais expressivos nos pacientes portadores de doença de Crohn, e o sobrepeso e obesidade mais frequentes na retocolite ulcerativa. A avaliação nutricional detalhada deve ser realizada periodicamente em todas as crianças e adolescentes portadores de doenças inflamatórias intestinais, combinada com diferentes métodos e recursos multidisciplinares. São necessários mais estudos na faixa etária pediátrica com populações mais numerosas. ${ }^{4}$

\section{ACOMETIMENTO RENAL}

A prevalência de nefrolitíase em pacientes com DII é maior do que na população geral, variando de $12 \%$ para $28 \%$, especialmente em pacientes com DII que tenham sido submetidos a procedimentos cirúrgicos do intestino como colectomia total com ileostomia, ressecção de intestino ou desvio intestinal. Diarreia e má absorção, muitas vezes descritas em pacientes de DII, são fatores de risco para a formação de cálculos renais. Glomerulonefrite é uma forma de envolvimento renal em pacientes com DII (DC e RCU). Têm sido descritos diferentes padrões histológicos de glomerulonefrite em DII: nefropatia por $\operatorname{IgA}$, IgM, membranosa e mesangiocapilar focal da membrana basal segmentar e anti glomerular. Glomerulonefrite estaria ligada diretamente a atividade da doença intestinal e melhora da função renal após remissão da inflamação intestinal tem sido demonstrado.

Nefrite tubulointersticial (NTI) também tem sido relatado em pacientes com DII. Embora a maioria dos casos têm sido associados com 5-aminosalicylate (5-ASA), ciclosporina A e exposição de inibidor do tumor necrose fator $\alpha$ (TNF $\alpha$ ), muitas vezes é difícil estabelecer se disfunção renal pode ser considerada uma manifestação extra intestinal ou se é devido a tratamento médico. No entanto, alguns trabalhos recentes têm destacado a ligação entre danos tubulointersticial e atividade da DII. Amiloidose secundária é uma complicação rara, mas significativa de DII que pode influenciar o prognóstico ainda mais do que a doença subjacente. ${ }^{37}$

\section{OUTROS}

Tromboembolismo venoso está relacionado a atividade da DII, embora não estejam estabelecidos protocolos para 
prevenção em pacientes com DII hospitalizados e o risco de um evento primário seja menor que em adultos, a profilaxia deve ser considerada em qualquer criança hospitalizada com DII grave, bem como em pacientes com fatores de risco, como acesso venoso central, história familiar ou imobilização. Além disso, as medidas não-farmacológicas devem ser adotadas em todos os pacientes hospitalizados..$^{38}$

\section{REFERÊNCIAS}

1. Bousvaros A, Sylvester F, Kugathasan S, Szigethy E, Fiocchi C. Challenges in pediatric inflammatory bowel disease. Inflamm Bowel Dis. 2006;12(9):885-913.

2. D'auria JP, Kelly M. Inflammatory bowel disease: top resources for children, adolescents, and their families. J Pediatr Health Care. 2013;27:e25-8.

3. Kane SV. IBD self-management. Bethesda (MD): AGA Press; 2010.

4. Santos GM, Silva LR, Santana GO. Repercussões nutricionais em crianças e adolescentes na presença de doenças inflamatórias intestinais. Revista Paulista de Pediatria. 2014;32:403-11.

5. Benchimol EI, Manuel DG, Guttmann A, Nguyen GC, Mojaverian $\mathrm{N}$, Quach P, et al. Changing age demographics of inflammatory bowel disease in Ontario, Canada: a population-based cohort study of epidemiology trends. Inflamm Bowel Dis. 201420(10):1761-9.

6. Kelsen J, Baldassano RN. Inflammatory bowel disease: the difference between children and adults. Inflamm. Bowel. Dis. 2008;14 (Supl 2):S9-11.

7. Vernier-Massouille G, Balde M, Salleron J, Turck D, Dupas JL, Mouterde O, et al. Natural history of pediatric Crohn's disease: a population-based cohort study. Gastroenterology. 2008;135(4):1106.

8. Van Limbergen J, Russell RK, Drummond HE, Aldhous MC, Round NK, Nimmo ER. Definition of phenotypic characteristics of childhood-onset inflammatory bowel disease. Gastroenterology. 2008;135(4):1114.

9. Kronman MP, Zaoutis TE, Haynes K, Feng R, Coffin SE. Antibiotic exposure and IBD development among children: a population-based cohort study. Pediatrics. 2012;130(4):e794-e803.

10. Radon K, Windstetter D, Poluda AL, Mueller B, von Mutius E, Koletzko S. Contact with farm animals in early life and juvenile inflammatory bowel disease: a case-control study. Pediatrics. 2007;120(2):354-61.

11. Ananthakrishnan AN, Khalili H, Konijeti GG, Higuchi LM, Silva P, Korzenik JR, et al. A prospective study of long-term intake of dietary fiber and risk of Crohn's disease and ulcerative colitis. Gastroenterology. 2013;145:970-7.

12. Ananthakrishnan AN, Khalili H, Higuchi LM, Bao Y, Korzenik JR, Giovannucci EL, et al. Higher predicted vitamin D status is associated with reduced risk of Crohn's disease. Gastroenterology. 2012;142(3):482-9.

13. Hou JK, Abraham B, El-Serag H. Dietary intake and risk of

\section{CONCLUSÕES}

Considerando o impacto na qualidade de vida e morbimortalidade dos pacientes portadores de doença inflamatória intestinal e as repercussões não apenas a nível de trato digestivo, dá-se a importância do médico assistente em valorizar as manifestações extra-intestinais, que podem inclusive surgir antes das manifestações intestinais.

developing inflammatory bowel disease: a systematic review of the literature. Am J Gastroenterol. 2011;106(4):563-73.

14. Cho JH. The genetics and immunopathogenesis of inflammatory bowel disease. Nat Rev Immunol. 2008;8:458.

15. Camus M, Esses S, Pariente B, Le Bourhis L, Douay C, Chardiny $\mathrm{V}$, et al. Oligoclonal expansions of mucosal T cells in Crohn's disease predominate in NKG2D-expressing CD4 T cells. Mucosal Immunol. 2014;7(2):325-34.

16. Bain CC, Scott CL, Uronen-Hansson H, Gudjonsson S, Jenson $\mathrm{O}$, Grip $\mathrm{O}$, et al. Resident and pro-inflammatory macrophages in the colon represent alternative context-dependent fates of the same Ly6Chi monocyte precursors. Mucosal Immunol. 2013;6(3):498510.

17. Neurath MF. Cytokines in inflammatory bowel disease. Nat Rev Immunol. 2014;14(5):329-42.

18. Jostins L, Ripke S, Weersma RK, Duerr RH, McGovern DP, Hui KY. Host-microbe interactions have shaped the genetic architecture of inflammatory bowel disease. Nature. 2012;491(7422):119-24.

19. Haberman Y, Tickle TL, Dexheimer PJ, Kim MO, Tang D, Karns R. Pediatric Crohn disease patients exhibit specific ileal transcriptome and microbiome signature. J Clin Invest. 2014;124(8):3617-33.

20. Hyams J, Markowitz J, Otley A, Rosh J, Mack D, Bousvaros A. Evaluation of the pediatric Crohn disease activity index: a prospective multicenter experience. J Pediatr Gastroenterol Nutr. 2005;41:416-21.

21. Lima MM, Silva LR, Franca RC, Santana GO, Ribeiro IT. Perfil de pacientes pediátricos com doenças inflamatórias intestinais, atendidos em ambulatório de referência na Cidade do Salvador. Rev Ciênc Méd Biol. 2013;12(3):337-43.

22. Kelsen J, Baldassano RN. Inflammatory bowel disease: the difference between children and adults. Inflamm Bowel Dis. 2008;14(Sup1 2):S9-11.

23. Silverberg MS, Satsangi J, Ahmad T, Arnott ID, Bernstein $\mathrm{CN}$, Brant SR, et al. Toward an integrated clinical, molecular and serological classification of inflammatory bowel diseases report of a Working Party of the 2005 Montreal World Congress of Gastroenterology. Can J Gastroenterol. 2005;19 (Supl A):5A-36A.

24. Haas-Beckert B, Heyman MB. Inflamaory bowel disease. In: Allen PJ, Vessey JA, Schapiro NA, editores. Primary care of the children with a chronic condition. 5. ed. St Louis (MO): Mobsy, 2009. p. $562-586$. 
25. Rodrigues M. Diagnóstico da doença inflamatória intestinal na criança e adolescente. International Journal of Nutrology. 2017;10(1):281.

26. Jose FA, Garnett EA, Vittinghoff E, Ferry GD, Winter HS, Baldassano RN, et al. Development of extra intestinal manifestations in pediatric patients with inflammatory bowel disease. Inflamm Bowel Dis. 2009;15(1):63.

27. Dotson JL, Hyams JS, Markowitz J, LeLeiko NS, Mack DR, Evans JS. Extraintestinal manifestations of pediatric inflammatory bowel disease and their relation to disease type and severity. J Pediatr Gastroenterol Nutr. 2010;51(2):140-5.

28. Woo SB. Atlas de patologia oral. Rio de Janeiro: Elsevier; 2013.

29. Galbraith SS, Drolet BA, Kugathasan S, Paller AS, Esterly NB. Asymptomatic inflammatory bowel disease presenting with mucocutaneous findings. Pediatrics. 2005;116(3):e439-44.

30. Farhi D, Cosnes J, Zizi N, Chosidow O, Seksik P, Beaugerie L, et al. Significance of erythema nodosum and pyoderma gangrenosum in inflammatory bowel diseases: a cohort study of 2402 patients. Medicine (Baltimore). 2008;87(5):281.

31. Parentin F, Nider S, Rassu N, Martelossi S, Ventura A, Naviglio S. Ocular Involvement in children with inflammatory bowel disease. Inflamm Bowel Dis. 2017;23(6):986-90.

32. Felekis T, Katsanos K, Kitsanou M, Trakos N, Theopistos V, Christodoulou D, et al. Spectrum and frequency of ophthalmologic manifestations in patients with inflammatory bowel disease: a prospective single-center study. Inflamm Bowel Dis. 2009;15(1):2934.

33. Yilmaz S, Aydemir E, Maden A, Unsal B. The prevalence of ocular involvement in patients with inflammatory bowel disease. Int J Colorectal Dis. 2007;22:1027-30.

34. Deneau M, Jensen MK, Holmen J, Williams MS, Book LS, Guthery SL. Primary sclerosing cholangitis, autoimmune hepatitis, and overlap in utah children: epidemiology and natural history. Hepatology. 2013;58(4):1392-400.

35. Wiskin AE, Wootton SA, Hunt TM, Cornelius VR, Afzal NA, Jackson AA, et al. Body composition in childhood inflammatory bowel disease. Clin Nutr. 2011;30(1):112-5.

36. Prince A, Whelan K, Moosa A, Lomer MC, Reidlinger DP. Nutritional problems in inflammatory bowel disease: the patient perspective. J Crohns Colitis. 2011;5:443-50.

37. Corica D, Romano CJ. Renal involvement in inflammatory bowel diseases. J Crohns Colitis. 2016;10(2):226-35.

38. Zitomersky NL, Levine AE, Atkinson BJ, Harney KM, Verhave $\mathrm{M}$, Bousvaros A, et al. Risk factors, morbidity, and treatment of thrombosis in children and young adults with active inflammatory bowel disease. J Pediatr Gastroenterol Nutr. 2013;57(3):343-7.

\section{Como citar:}

Marques ML, Patrício MP. Manifestações extra intestinais de espectros da doença inflamatória intestinal em crianças e adolescentes: artigo de revisão. Rev Med UFC. 2019 jan-mar;59(1):44-52. 